\title{
Erratum to: A doubly sparse approach for group variable selection
}

\author{
Sunghoon Kwon ${ }^{1}$ - Jeongyoun $\mathrm{Ahn}^{2}$. Woncheol Jang ${ }^{3}$. \\ Sangin $\mathrm{Le}^{4}$. Yongdai Kim ${ }^{3}$
}

(C) The Institute of Statistical Mathematics, Tokyo 2017

\section{Erratum to: Ann Inst Stat Math DOI 10.1007/s10463-016-0571-z}

Unfortunately, original article has been published without revised date.

Revised date should be: 5 January 2016.

The online version of the original article can be found under doi:10.1007/s10463-016-0571-z.

$凶$ Yongdai Kim

ydkim0903@gmail.com

1 Department of Applied Statistics, Konkuk University, 120 Neungdong-ro, Gwangjin-gu, Seoul 05029, Korea

2 Department of Statistics, University of Georgia, Athens, GA 30602, USA

3 Department of Statistics, Seoul National University, 1 Gwanak-ro, Gwanak-gu, Seoul 08826, Korea

4 Quantitative Biomedical Research Center, University of Texas Southwestern Medical Center, Dallas, TX 75390, USA 